\title{
Two-Component Density Functional Study of Positron-Vacancy Interaction in Metals and Semiconductors
}

\author{
S. ISHIBASHI* \\ Research Center for Computational Design of Advanced Functional Materials (CD-FMat), AIST, \\ 1-1-1 Umezono, Tsukuba, Ibaraki 305-8568, Japan
}

\begin{abstract}
The positron-vacancy interaction in metals and semiconductors has been studied by the two-component density-functional-theory formalism. If a positron is not trapped at the vacancy, atoms surrounding the vacancy show inward relaxation for most cases except for nitrides, where outward relaxation is observed. The presence of positron suppresses the inward relaxation. Positron lifetimes have been calculated with and without the positron effect. The difference between two lifetime values is investigated in relation to the bulk modulus.
\end{abstract}

DOI: 10.12693/APhysPolA.132.1602

PACS/topics: 71.60.+z, 78.70.Bj, 61.72.jd

\section{Introduction}

The positron can be utilized as a powerful probe for detecting vacancy-type defects in various solids, since positrons are selectively trapped at (cation) vacancies in metals and semiconductors [1-3]. The momentum distribution of positron-annihilation radiation and the positron lifetime reflect the local environment of the annihilation site. This makes it possible to detect defects and to distinguish them. In practical cases, theoretical predictions are often crucial in interpreting experimental results and in identifying defect species. To describe the positron state in solids, where many nuclei and electrons exist, the two-component density-functional-theory formalism was introduced $[4,5]$. Boroński and Nieminen derived explicit expressions for the electron-positron correlation-energy functional and proposed the fully selfconsistent scheme [6] (described as "two-component (TC) scheme" hereafter). In many practical calculations, a simplification is made assuming that the positron affects neither the electronic structure nor the atomic arrangement. This simplified scheme is called "conventional (CV) scheme". To describe the delocalized positron state in a bulk, the CV scheme is appropriate.

So far, there have been a limited number of applications of the (full) TC scheme in calculating positron states trapped at defects. One reason is that the computational cost for the TC scheme is approximately 10 times higher than that for the $\mathrm{CV}$ scheme. Another reason is that, in many cases, the CV scheme and the TC scheme give similar annihilation parameters (Doppler broadening spectra and positron lifetimes) because of the feedback effect [7].

Recently, we reported calculated results of Dopplerbroadening spectra and positron lifetimes using the two schemes for divacancies in $\mathrm{C}, \mathrm{Si}, \mathrm{Ge}, \mathrm{SiC}, \mathrm{AlN}, \mathrm{GaN}$

*corresponding author; e-mail: shoji.ishibashi@aist.go.jp and $\mathrm{InN}$ and found that the difference between the two schemes depends on the bulk modulus [8]. For the relatively soft materials $\mathrm{Si}$ and Ge, the difference is significant. Subsequently, the positron-monovacancy interaction in $d$-block metals (except for $\mathrm{Mn}, \mathrm{Tc}$, and $\mathrm{Hg}$ ) was investigated [9]. When the atomic positions are relaxed neglecting the positron effect, for most cases, inward relaxation of the atoms surrounding the vacancy occurs, especially for the group V metals. The inward relaxation is suppressed when the positron effect is taken into account. As a result, the positron lifetime calculated neglecting the positron effect is generally shorter than that obtained considering the positron effect.

In the present paper, extending the previous studies mentioned above, a computational study of the positronvacancy interaction in $\mathrm{Mg}$ and $\mathrm{Al}$ as well as in III-V zinc-blende (ZB) semiconductors has been performed. As for AlN, GaN and InN, they usually have the wurtzite (WZ) structure. Calculations were made on their WZ structures also for comparison. Positron states and lifetimes have been calculated by the CV scheme and the TC scheme with and without structural relaxation.

\section{Computational method}

All the calculations have been performed with our computational code QMAS (Quantum MAterials Simulator) $[10,11]$. In the conventional scheme, electronic structures and atomic positions are calculated independently of the presence of the positron. On the other hand, in the TC scheme, electronic structures and atomic positions are affected by the presence of the positron through the electrostatic energy and the positron-electron-correlation energy $[6,7]$. Electronic wave functions were described with the plane-wave basis in the framework of the projector augmented-wave (PAW) method [12]. For the electronic exchange-correlation energy, the Perdew-BurkeErnzerhof (PBE) functional of the generalized gradient approximation (GGA) [13] was used. Calculations were performed on supercells with the size of $3 \times 3 \times 3$ for FCC (108- 1 atoms), $4 \times 4 \times 4$ for BCC (128- 1 atoms), or 
$3 \times 3 \times 3$ for ZB (216-1 atoms). As for HCP and WZ cases, an orthorhombic supercell equivalent to $4 \times 4 \times 2$ primitive unit cells was constructed for each. In the supercell, there are 64-1 atoms for HCP while there are 128-1 atoms for WZ. We used experimental lattice parameters and, for semiconductors, experimental high-frequency dielectric constants. The number of $\boldsymbol{k}$ points is 8 for metals while 1 ( $\Gamma$ point) for semiconductors. Further details including the computational procedure for the TC scheme are described in Refs. [8, 9].

\section{Results and discussion}

For each metal and scheme (CV or TC), the change in the average distance $\Delta d$ of the first-nearest-neighbor atoms ( 6 for HCP, 12 for FCC, 8 for BCC) from the vacancy center were evaluated. The results including those for the $d$-block metals (except for $\mathrm{Mn}, \mathrm{Tc}$, and $\mathrm{Hg}$ ) [9] are plotted in Fig. 1 as a function of the atomic number $Z$. For HCP metals, values for the six second-nearestneighbor atoms are also plotted. Similarly to most $d$ block metals, vacancies in $\mathrm{Mg}$ and $\mathrm{Al}$ show inward relaxations when the positron effect is neglected (CV). If the positron effect is considered (TC), slight outward relaxations are observed. Makkonen and Puska reported structural relaxations around vacancies for several representative materials [14]. Although a direct comparison is difficult due to different computational conditions (the electronic exchange-correlation, etc.), the present results for metals show a similar qualitative tendency to their previous results. They pointed out that the total energy curve is very flat over a large range of ionic relaxation. In such a case, subtle changes in the computational conditions may result in different degrees of structural relaxation.

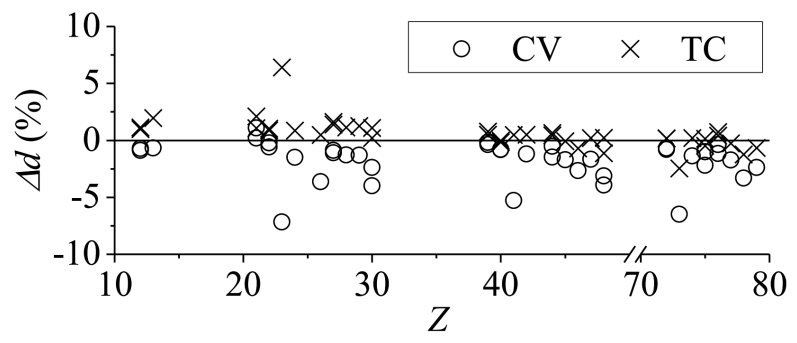

Fig. 1. $Z$-dependence of the change in average distance $\Delta d$ of the first-nearest-neighbor atoms from the vacancy center after atomic-position relaxation. For HCP metals, values for the second-nearest-neighbor atoms are also plotted. The results for $d$-block metals are taken from Ref. [9].

Figure 2 represents calculated positron lifetime $\tau$ values for the two schemes (CV and TC) with no relaxation (NR) or relaxation (RL). For both $\mathrm{Mg}$ and $\mathrm{Al}$, even on the unrelaxed structures, the positron lifetime values for the TC scheme are longer than those for the CV scheme. As already mentioned in Ref. [9], it is due to lower enhancement factors in the TC scheme. The differences

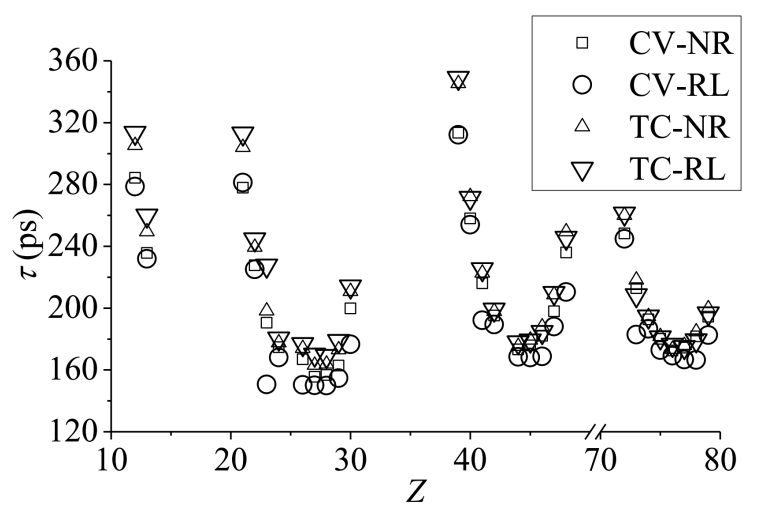

Fig. 2. Z Z-dependence of the calculated positron lifetime $\tau$ trapped at a vacancy. The results for $d$-block metals are taken from Ref. [9].

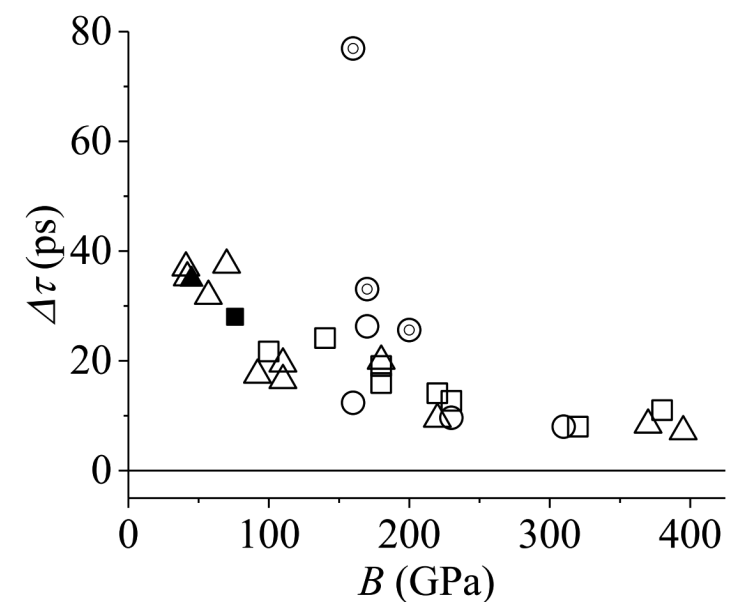

Fig. 3. Difference between positron lifetimes $\Delta \tau$ calculated by the CV and TC schemes with structural relaxation as a function of bulk modulus $B$. Triangles, squares, and circles correspond to HCP, FCC, and BCC metals, respectively. Filled symbols correspond to $\mathrm{Mg}$ and Al. The results for the group V metals are shown by double circles. The results for $d$-block metals are taken from Ref. [9].

between positron lifetimes $\Delta \tau$ calculated by the $\mathrm{CV}$ and TC schemes with structural relaxation for $\mathrm{Mg}$ and $\mathrm{Al}$ are plotted together with the results for $d$-block metals in Fig. 3 as a function of bulk modulus $B$. The results for $\mathrm{Mg}$ and $\mathrm{Al}$ follow a general trend for the $d$-block metals except for the group $\mathrm{V}$ metals, which show an extraordinary behavior.

The $\Delta d$ values for a cation vacancy in III-V ZB semiconductors are plotted in Fig. 4. For InAs, no welllocalized positron state was obtained. Since the TC scheme is appropriate only for well-localized positron states, the TC result for InAs is not shown. Except for nitrides and BP, similarly to most metals, inward relaxations are observed. The inward relaxation tends to increase with the row of the periodic table in which the 


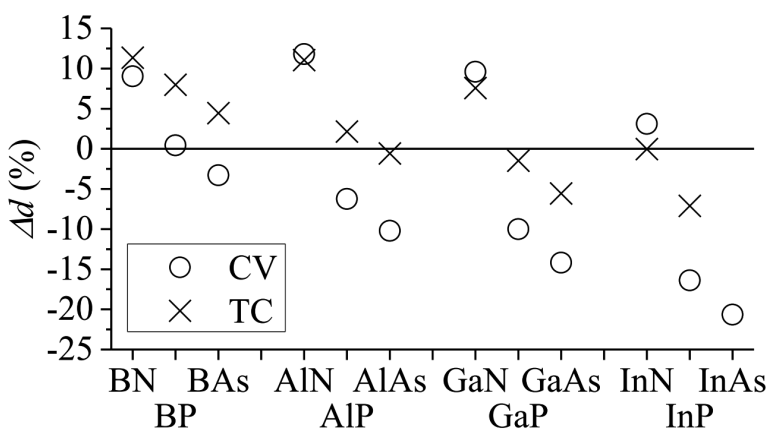

Fig. 4. Change in average distance $\Delta d$ of the firstnearest-neighbor atoms from the vacancy center after atomic-position relaxation for a cation vacancy in III-V ZB semiconductors.

TABLE I

Comparison of $\Delta d$ and $\tau$ for $\mathrm{ZB}$ and WZ nitrides.

\begin{tabular}{cc|c|c|c|c|c|c}
\hline \hline & & \multicolumn{2}{|c|}{$\Delta d[\%]$} & \multicolumn{4}{c}{$\tau[\mathrm{ps}]$} \\
\cline { 3 - 8 } & & CV & TC & CV-NR & CV-RL & TC-NR & TC-RL \\
\hline \multirow{2}{*}{ AlN } & ZB & 11.81 & 11.04 & 200.8 & 228.4 & 201.7 & 233.9 \\
& WZ & 10.55 & 9.68 & 199.8 & 223.8 & 200.4 & 227.9 \\
\multirow{5}{*}{ GaN } & ZB & 9.61 & 7.58 & 209.0 & 229.2 & 211.8 & 232.8 \\
& WZ & 7.51 & 6.07 & 206.8 & 222.0 & 208.8 & 225.3 \\
InN & ZB & 3.11 & -0.03 & 245.8 & 250.3 & 253.8 & 251.7 \\
& WZ & 2.82 & 0.02 & 244.0 & 247.3 & 251.7 & 249.7 \\
& & & & & & &
\end{tabular}

constituent elements are located. As for the outward relaxation observed in the $\mathrm{CV}$ case for nitrides $(\mathrm{BN}, \mathrm{AlN}$, $\mathrm{GaN}$ and InN), it can be ascribed to the Coulombic repulsion among the anion atoms as Mizuno et al. speculated for oxides [15]. For AlN, GaN, and InN, the outward relaxation is suppressed by the presence of a positron. In Table I, the results for three WZ nitrides are listed and compared with the ZB results. Except for the case of "InN TC", each value of $\Delta d$ for ZB is marginally larger than the corresponding $\mathrm{WZ}$ value.

Figure 5 represents calculated positron lifetime $\tau$ values for the cation vacancy in the III-V ZB semiconductors. There seems a systematic trend depending on the position in the periodic table. For each compound except for the nitrides, a significant difference between the CVRL and TC-RL results is observed. The results for the WZ nitrides are listed and compared with the $\mathrm{ZB}$ results in Table I. Each $\tau$ value for ZB is slightly longer than the corresponding $\mathrm{WZ}$ value. The $\Delta \tau$ values for the cation vacancy in the III-V ZB semiconductors are plotted as a function of $B$ in Fig. 6. Although the relationship between $\Delta \tau$ and $B$ is obscure compared with the metal case, $\Delta \tau$ tends to be large for low- $B$ compounds.

In the present calculations, valence and core electrons are not distinguished in evaluating the enhancement factor and the positron-electron-correlation energy. For semiconductors, the high-frequency dielectric constant for the bulk is used to describe the less screening even in treating trapped states at vacancies. Perhaps, these

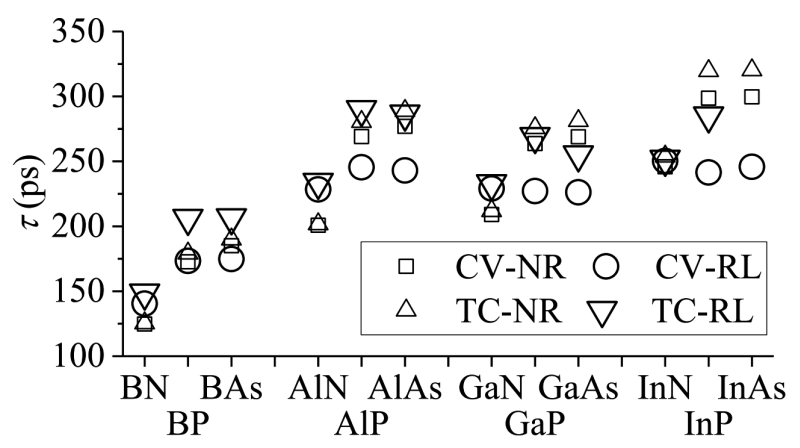

Fig. 5. Calculated positron lifetime $\tau$ trapped at a cation vacancy in III-V ZB semiconductors.

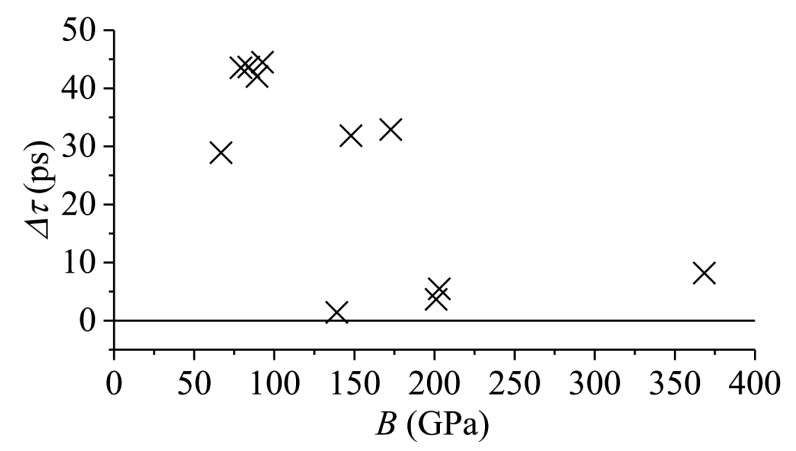

Fig. 6. Difference between positron lifetimes $\Delta \tau$ calculated by the CV and TC schemes with structural relaxation for a cation vacancy in III-V ZB semiconductors as a function of bulk modulus $B$.

are oversimplifications. To remove these drawbacks, one possible solution is applying the GGA to the positronrelated terms in the calculation $[16,17]$. Unfortunately, a TC version of the positron GGA has not yet been established. Developments in this field is awaited.

\section{Conclusion}

The positron-vacancy interaction in metals and semiconductors has been studied by the two-component density-functional-theory formalism. If the positron effect is neglected, except for nitrides where the Coulombic repulsion among the anion atoms surrounding the vacancy is strong, inward relaxation is observed for each material. The inward relaxation is suppressed when the positron effect is taken into account. For a low- $B$ material, there is a significant difference in calculated positron lifetime depending on whether the positron effect exists. Calculations by the two-component DFT method are desirable for such a case.

\section{Acknowledgments}

The author is grateful to Dr. Masanori Kohyama, Professor Akira Uedono, and Professor Kiyoyuki Terakura for useful information and stimulating discussions. This work was partly supported by JSPS KAKENHI 
Grant Number JP16H06424 and by the Cross-Ministerial Strategic Innovation Promotion Program - Unit D66 - Innovative Measurement and Analysis for Structural Materials (SIP-IMASM), operated by the Cabinet Office, Japan.

\section{References}

[1] M. J. Puska, R.M. Nieminen, Rev. Mod. Phys. 66 841 (1994).

[2] R. Krause-Rehberg, H.S. Leipner, Positron Annihilation in Semiconductors, Springer, Berlin 1999.

[3] F. Tuomisto, I. Makkonen, Rev. Mod. Phys. 85, 1583 (2013).

[4] B. Chakraborty, Phys. Rev. B 24, 7423 (1981).

[5] B. Chakraborty, R.W. Siegel, Phys. Rev. B 27, 4535 (1983).

[6] E. Boroński, R.M. Nieminen, Phys. Rev. B 34, 3820 (1986).

[7] M.J. Puska, A.P. Seitsonen, R.M. Nieminen, Phys. Rev. B 52, 10947 (1995).
[8] S. Ishibashi, A. Uedono, J. Phys.: Conf. Ser. 674, 012020 (2016).

[9] S. Ishibashi, J. Phys. Soc. Jpn. 84, 083703 (2015).

[10] S. Ishibashi, T. Tamura, S. Tanaka, M. Kohyama, K. Terakura, Phys. Rev. B 76, 153310 (2007).

[11] S. Ishibashi, A. Uedono, J. Phys.: Conf. Ser. 505, 012010 (2014).

[12] P. Blöchl, Phys. Rev. B 50, 17953 (1994).

[13] J.P. Perdew, K. Burke, M. Ernzerhof, Phys. Rev. Lett. 77, 3865 (1996).

[14] I. Makkonen, M.J. Puska, Phys. Rev. B 76, 054119 (2007).

[15] M. Mizuno, H. Araki, Y. Shirai, Mater. Trans. 45, 1964 (2004)

[16] B. Barbiellini, M.J. Puska, T. Torsti, R.M. Nieminen, Phys. Rev. B 51, 7341 (1995).

[17] B. Barbiellini, J. Kuriplach, Phys. Rev. Lett. 114, 147401 (2015). 